\title{
Induced Desynaptic Male Sterile Lines in Soybean
}

\author{
Girjesh Kumar ${ }^{1}$ and Priyanka Rai ${ }^{2}$ \\ Plant Genetics Laboratory, Department of Botany, University of Allahabad, Allahabad-211002, U.P., India
}

Received July 10, 2006; accepted September 12, 2006

\begin{abstract}
Summary In a study on soybean using gamma rays, we came across with some sterile mutants. Cytological studies of these mutants clearly revealed desynapsis in them. In these sterile plants only few bivalents and a high frequency of univalents have been recorded and they displayed a very high percentage of pollen sterility. Later meiotic stages were also found to be highly disturbed. The plants were identified as male sterile ones as few pods were formed. It might be possible that gamma rays have acted on some genes responsible for synapsis and chiasma formation and resulted in early chiasmate dissociation suggesting that gamma rays can act as a potential tool in the development of male sterile lines. Study of desynaptic mutants is a potentially important source of information on the chiasma maintenance mechanism. These mutants furthermore can provide useful cytological and genetical informations on the male sterility occured in higher plants.
\end{abstract}

Key words Soybean, Desynapsis, Chromosomal abnormalities, Male-sterility, Gamma-rays.

Meiosis occupies a central role in the life cycle of all sexually reproducing eukaryotes, and it is through this process that eukaryotes achieve chromosome reduction, ordered chromosome segregation into haploid products and recombination of chromosomes. This involves a complex orchestration of events including chromosome pairing at zygotene, genetic exchange at pachytene, chiasmata formation at diplotene and chromosome segregation at anaphase I and II (Franklin et al. 1999). At least one chiasmata per bivalent is essential for orderly disjunction, otherwise, some homologues may migrate to same pole and form aneuploid gametes. The pairing and recombination of chromosome is under genetic control (Rees 1961). A mutation in the genes controlling the meiotic recombination may lead to failure or early dissolution or reduction of chiasma formation. According to Gottschalk and Kleine (1976), chromosome pairing is under the control of 2 groups of genes designated as and $d s$ which when present in recessive state, cause pairing to fail. The absence or failure of synapsis is termed as asynapsis, while the immediate separation of the homologues following normal pachytene pairing is specified as desynapsis (Gottschalk and Kaul, 1980a, b).

Desynapsis has been studied in various plants like Pennisetum (Jauher et al. 1971), Zinnia (Singh and Gupta, 1981), Chilli (Rao and Kumar, 1983), Barley (Srivastava 1974, Kumar and Singh 2002), Rice (Reddi and Rao 2000), Chickpea (Kumar and Sharma, 2001), Anogeissus (Rao and Kumar 2003) and Soybean (Palmer and Horner 2000, Bione et al. 2002b).

In a mutation-breeding programme on soybean (Glycine max) through $\gamma$-rays we came across with some sterile mutants. Cytogenetic analysis of these sterile mutants clearly revealed the occurrence of desynapsis in these plants. The present study is an attempt to understand the genetic behavior of sterility as well as its mechanism through cytogenetic analysis of these sterile-desynaptic mutants as our knowledge on these aspects is still very limited especially in case of soybean, although some very interesting facts have already been reported.

* Corresponding author, e-mail: ${ }^{1}$ kumar_girjesh @yahoo.com, ${ }^{2}$ priyankarai30@yahoo.co.in 


\section{Materials and methods}

Soybean seeds of var. JS 90-41 were obtained from National Research Center for Soybean, Indore and were irradiated at different doses of $\gamma$-rays viz. 100, 200, 300, 400 and 500 Gy. These seeds were then sown along with control seeds. At $400 \mathrm{~Gy}$ dose, some plants were detected which had very low frequency of pod formation. The seeds from these plants were then sown in the next generation along with normal seeds to raise the $\mathrm{M}_{2}$ generation. At the time of flowering young floral buds of appropriate size were fixed in ethanol and glacial acetic acid $(3: 1)$ solution after which they were transferred to $70 \%$ alcohol and stored at $4^{\circ} \mathrm{C}$. Slides were prepared using anther squash technique with $2 \%$ acetocarmine. Five plants were cytologically analyzed and pollen fertility of these plants was determined using acetocarmine glycerine stainability test.

\section{Observations}

Observation of $\mathrm{M}_{2}$ generation plants at $400 \mathrm{~Gy}$ dose of $\gamma$-irradiation revealed that 5 plants though morphologically normal and displayed normal flowering but the pod formation in them was negligible. These plants retained their leaves at maturity and were identified as male sterile according to the observation made by Bione et al. 2002a, b.

Cytological studies of the male sterile plants clearly revealed that these plants had an abnormal meiotic behavior and were found to be desynaptic. The meiosis in control plants was found to be normal (Figs.1-3) with high pollen fertility (approximately 96\%). In the fertile plants furthermore, twenty bivalents $(2 n=40)$ with 1 or 2 terminal or sub-terminal chiasmata were observed at diakinesis and metaphase I. While in the sterile plants only few bivalents and a high frequency of univalents have been recorded (Table 1) (Figs. 4-7). These desynaptics displayed a high percentage of pollen sterility, which was recorded to be $88.35 \%, 86.52 \%, 93.29 \%, 91.76 \%$ and $90.07 \%$ in plant number 1, 2, 3, 4 and 5, respectively (Table 2).

In the desynaptics, the univalents were not found independently but these tend to show a mutual dependence of position. Here, univalents belonging to the same pair were preferably found arranged closely to each other. In some of the cases 40 univalents were seen at diakinesis and metaphase I.

Meiosis in fertile plants proceeded normally with 20 bivalents arranged at metaphase plate (Fig. 2) and separated equally and later moved to opposite poles at anaphase I (Fig. 3).

In the desynaptic mutants, univalents were predominantly present at diakinesis and metaphase I stages. Sister chromatid cohesion was also not found to be perfect and some of the sister chromatids could not maintain attachment up to anaphase II and separated at an early stage of the division. It was evident as more than 40 chromosome complements were observed at diakinesis (Fig. 4) and metaphase I in some cases. At metaphase I, bivalents and univalents congregated at the equatorial plate but the chromosome segregation was not perfect. As a consequence of univalent formation the succeeding meiotic stages were found to be highly irregular (Table 2). At anaphase I, unequal separation of chromosomes (Fig. 8) was very common. Besides it, anaphase I showed variable number of laggards, late separation, bridges, disturbed polarity and tripolarity etc.

Since the bivalents had 1 or 2 chiasmata, delay in chiasma terminalization promoted the occurrence of laggards at anaphase I. Due to the irregular distribution of chromosomes at anphase I, those that reach the poles were organized in dyad nuclei and the lagging chromosomes developed into micronuclei at telophase I.

The second meiotic division in the desynaptic mutants also exhibited drastic meiotic irregularities in majority of PMCs (Figs. 9-12) as recorded in the 1st division. More commonly dyads contained unequal number of chromosomes on the 2 spindles and sometimes 3 metaphase plates were seen at metaphase II (Fig. 9) arising as a result of tripolar spindle formation at metaphase I. Chro- 

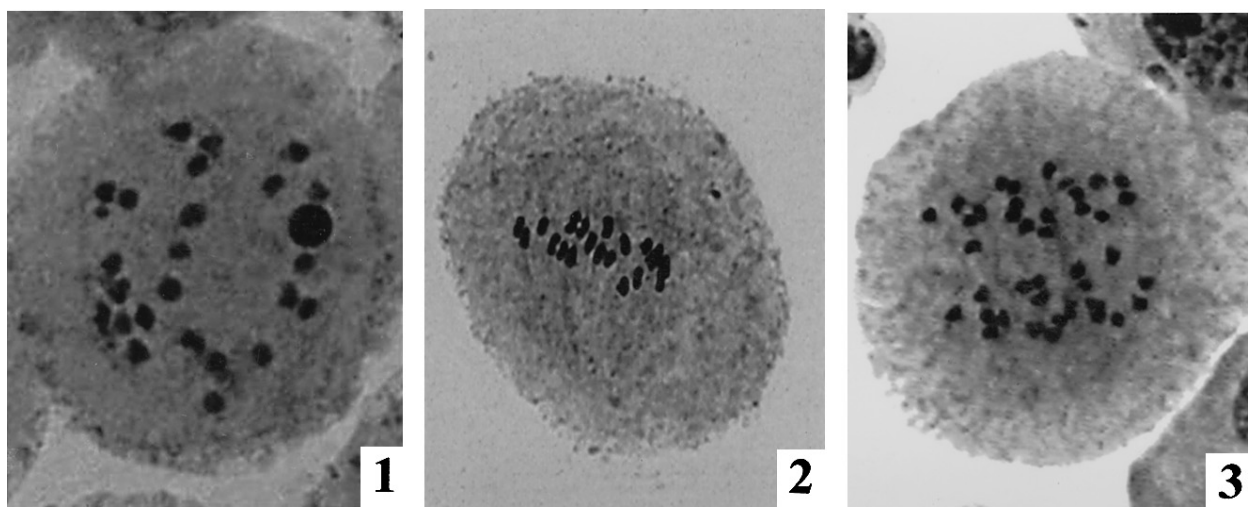

Fig. 1-3. Meiotic stages in auther squash of control plants. 1: Diakinesis $(n=20), 2$ : Metaphase I (with 20 bivalents at equator), 3: Early Anaphase I (with $20: 20$ separation).

Table 1. Bivalent and univalent configuration at diakinesis/metaphase I in 5 induced desynsptics

\begin{tabular}{|c|c|c|c|c|c|c|}
\hline \multirow{2}{*}{$\begin{array}{l}\text { Plant } \\
\text { No. }\end{array}$} & \multirow{2}{*}{$\begin{array}{c}\text { Total } \\
\text { PMCs } \\
\text { observed }\end{array}$} & \multicolumn{5}{|c|}{ Diakinesis/Metaphase I configuration } \\
\hline & & $\begin{array}{l}20 \mathrm{II} \\
(\%)\end{array}$ & $\begin{array}{c}(15-19) I I+(10-2) I \\
(\%)\end{array}$ & $\begin{array}{c}(10-14) \mathrm{II}+(20-12) \mathrm{I} \\
(\%)\end{array}$ & $\begin{array}{c}(5-9) I I+(30-22) \mathrm{I} \\
(\%)\end{array}$ & $\begin{array}{c}(0-4) \mathrm{II}+(40-33) \mathrm{I} \\
(\%)\end{array}$ \\
\hline 1 & 356 & 4.49 & 15.73 & 24.71 & 22.19 & 32.86 \\
\hline 2 & 324 & 5.55 & 13.53 & 28.70 & 29.93 & 22.22 \\
\hline 3 & 393 & 2.79 & 12.97 & 26.46 & 29.77 & 27.98 \\
\hline 4 & 412 & 3.64 & 23.78 & 19.66 & 24.75 & 28.15 \\
\hline 5 & 381 & 5.51 & 32.54 & 25.45 & 24.40 & 12.07 \\
\hline
\end{tabular}

mosome distribution at anaphase II was highly irregular with variable number of laggards and multipolarity (Fig. 10). Telophase II also showed laggards and more than 4 groups were observed in many PMCs. Sometimes the chromosomes strayed into the cytoplasm and failed to be included in the daughter nuclei and formed independent nuclei. Micronuclei ranging from 1-6 per tetrad were observed in most of the PMCs (Fig. 11). As a result variable number of microspore formation at tetrad stage was recorded from 3-7 microspores. Normally microsporocyte is divided into a quartet of cells, the nucleus of each containing a set of 20 chromosomes but here as a result of 2 irregular divisions (Meiosis I and Meiosis II) very few PMCs showed a tetrad of equal sized spores characteristic of normal. Microspores of varying sizes and numbers were also formed.

\section{Discussion}

The close proximity of homologous chromosomes (univalents) and arrangement of chromosomes at the metaphase plate suggested a very recent dissociation indicating that this plant belongs to the category of desynaptic type. Also, according to Peirson et al. (1997), in asynaptics, univalents never align at the equator at metaphase I, while in desynaptics, the bivalent as well as univalents congregate at metaphase plate. This also suggests that the present mutants are of desynaptic type.

Though desynapsis of spontaneous origin has been reported in number of plants, but induced desynaptic variations by physical or chemical mutagens are not many (Gottschalk and Kaul 1980a, b, Koduru and Rao 1981, Kumar and Sharma 2001, Kumar and Singh 2002). 

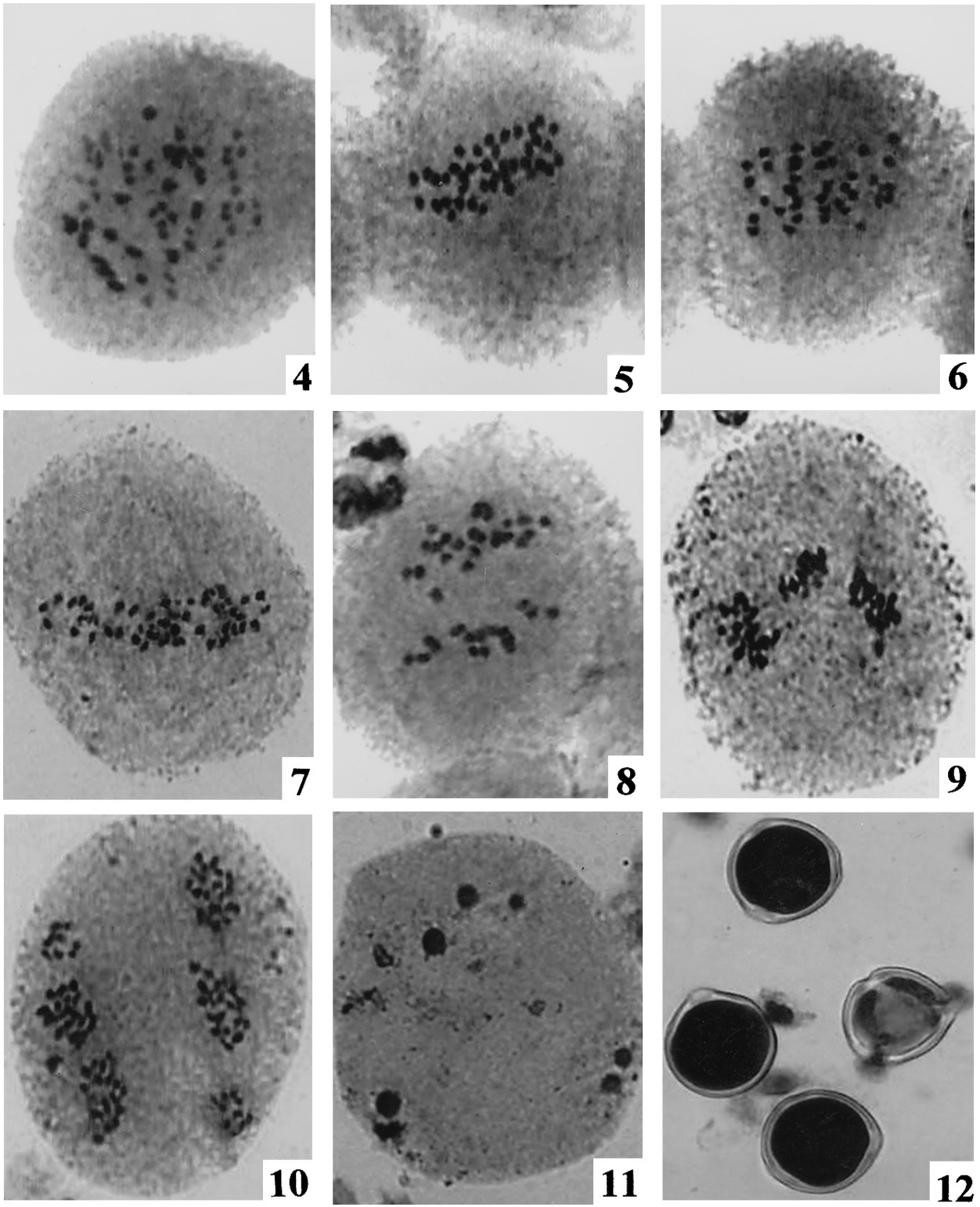

Fig. 4-12. Meiotic stages in anther squash of the desynaptic mutants. 4: Diakinesis $(3 \mathrm{II}+23 \mathrm{I}+22$ separated sister chromatids), 5: Metaphase I (7II+26I), 6: Metaphase I (4II+32I), 7: Metaphase I (34I+12 separated sister chromatids), 8: Unequal separation at Anaphase I (24:16), 9: Three Metaphase plates at Metaphase II, 10: Multipolarity at Anaphase II, 11: Micronuclei, 12: Pollen grains: Fertile (filled and darkly stained) and sterile (empty and lightly stained). 
Table 2. Percentage of PMCs with meiotic abnormalities in each phase of meiosis and pollen sterility

\begin{tabular}{|c|c|c|c|c|c|c|c|c|c|c|c|}
\hline \multirow{2}{*}{$\begin{array}{c}\text { Plant } \\
\text { No. }\end{array}$} & \multirow{2}{*}{$\begin{array}{c}\text { No. of PMCs } \\
\text { observed/ } \\
\text { plant }\end{array}$} & \multicolumn{8}{|c|}{ Phases of Meiosis (\% Abnormality) } & \multirow{2}{*}{$\begin{array}{c}\text { Mean } \\
\text { abnormality/ } \\
\text { plant (\%) }\end{array}$} & \multirow{2}{*}{$\begin{array}{c}\text { Pollen } \\
\text { sterility } \\
(\%)\end{array}$} \\
\hline & & Dia & Meta I & Ana I & Telo I & Meta II & Ana II & Telo II & Tetrad & & \\
\hline 1 & 647 & 97.70 & 97.64 & 94.44 & 90.90 & 80.24 & 82.14 & 78.94 & 81.17 & 87.88 & 88.35 \\
\hline 2 & 547 & 97.22 & 92.59 & 93.42 & 83.33 & 81.33 & 79.71 & 82.08 & 77.35 & 85.87 & 86.52 \\
\hline 3 & 575 & 100 & 98.66 & 100 & 94.20 & 88.73 & 87.34 & 78.57 & 81.66 & 91.14 & 93.29 \\
\hline 4 & 582 & 96.38 & 92.95 & 93.75 & 91.04 & 86.66 & 83.09 & 80.88 & 84.33 & 88.63 & 91.76 \\
\hline 5 & 613 & 95.18 & 93.67 & 92.53 & 85.71 & 82.66 & 80.76 & 76.92 & 83.13 & 86.32 & 90.07 \\
\hline
\end{tabular}

Dia; Diakinesis, Meta; Metaphase, Ana; Anaphase, Telo; Telophase.

A considerable variation in the number of univalents per PMC was observed. As this desynaptic condition was found to be single gene controlled, it appears that the differential expression of the same gene could be either due to the environmental factors or due to some modifying gene.

It is well known that the early meiotic events are most susceptible to change by intrinsic or extrinsic agents. The agent in the present case was $\gamma$-rays. It might have acted on some genes responsible for synapsis and chiasma formation and resulted in early chiasmate dissociation. Various theories have been forwarded by different workers from time to time to explain the occurrence of desynapsis. Maguire $(1993,1995)$ has emphasized that the recombination is insufficient to hold the chiasma in place. Additional factors, located either at the chiasmata or between sister chromatids are required to maintain chiasmata. According to Armstrong et al. (2002), the genes responsible for the formation of synaptinemal complex (SC) proteins have highly conserved sequence. A mutation in these genes might have lead to defective SC proteins, which are unable to hold the homologues together for long. Earlier workers like Sharma and Reinbergs (1974) proposed that recessive homozygous condition of $d s$ gene might cause chiasma to dissociate early. Similarly, Simchen and Stamberg (1969) concluded from their study that a mutation in highly conserved rec gene system might lead to failure of chiasma formation and recombination. Ji et al. (1999) proposed that recombination modifier mutation in rec gene might reduce recombination to a point where no pairing occurs.

Study of desynaptic mutants is a potentially important source of information on the chiasma maintenance mechanism. While, crossing-over is essential for chiasma formation, the maintenance of these chiasmata requires additional genetic factors. The latter seems to rely on the activity of the synaptonemal complex (SC) central region. Recent studies demonstrating that desynaptic plants have a defective SC which led Maguire et al. $(1991,1993)$ to suggest that the substance binding sister chromatids is derived from the SC. A multisubunit complex of conserved protein called cohesions is thought to provide longitudinal links along the chromatids and maintaining the chiasma in place (Bhatt et al. 1999, Nasmyth et al. 2000). Defects in synapsis not only result in recombination and chiasmata deficiency but might also hinder establishment of sister chromatid cohesion necessary for proper chromosome segregation (Bickel and Orr-Weaver 1996). And, probably this is the reason that not only the homologous chromosomes separated but some of the sister chromatids also were not able to hold together and separated at an early stage of division. The premature separation of sister chromatids is a rare phenomenon (Miyazaki and Orr-Weaver 1994) and in most of the cases the univalents maintain the sister chromatid cohesion until anaphase II for proper segregation. It might also be possible that some sort of mutations have occurred in the protein encoding genes required for sister chromatid cohesion which resulted in premature sepration of sister chromatids.

For the occurrence of other chromosomal anomalies in the desynaptic mutants, it can be concluded that the univalents might have distributed randomly to the daughter nuclei leading to unequal separation or unbalanced gametes or they were lost as micronuclei in the cytoplasm. The laggards and precocious chromosomes also contributed to the micronuclei. The univalents might also 
result in tripolar spindle at anaphase I and 3 metaphase plates at metaphase II since the unpaired chromosomes disrupt the spindle owing to their single functional kinetocore as suggested by Dawe and Cande (1995). The unequal distribution of chromosomes at anaphase might have resulted in the production of microspores of various sizes and ploidy levels that normally do not develop into viable pollen grains. The present mutants also displayed a very high pollen sterility ranging from $86.52 \%$ to a maximum of $93.29 \%$.

Due to the fact that most synaptic mutations also affected female fertility, Peirson et al. (1997) suggested that a set of genes controlled homologous chromosome pairing and recombination in both anthers and ovules. Most of the synaptic mutants (desynaptic as well as asynaptic) reported in soybean were found to be male sterile and also female sterile (Bione et al. 2002b) but, in our case these mutants produced few pods suggesting cross-pollination by insects, indicating that the mutation affected only microsporogenesis.

Prakken (1943) classified desynaptic mutants according to chromosome dissociation into weak, medium-strong and complete. Taking into consideration the frequency of bivalents found at diakinesis and metaphase I, all the 5 mutants can be classified as medium-strong type desynaptics.

On the basis of cytomorphological studies, it was worthnoting that all the desynaptic mutant were green at maturity, the sister chromatids separated at an early stage and few pods were also formed which suggests that the present mutants might represent a new mutation, different from all the previously reported ones in soybean. These mutants furthermore can provide useful cytological and genetical informations about the manner male sterility occurs in higher plants. Besides it, the study also shows the importance of $\gamma$-rays as potential tool for development of male steriles in soybean, which can be a big achievement as it is a strictly self-pollinated crop.

\section{Acknowledgement}

Authors are thankful to National Research Center for Soybean, Indore for providing seeds and National Botanical Research Institute, Lucknow for providing gamma irradiation facility. Sincere thanks are due to all the members of Plant Genetics Laboratory for valuable help.

\section{References}

Armstrong, S. J., Caryl, A. P., Jones, G. H. and Franklin, F. C. H. 2002. Asy 1, a protein required for meiotic chromosome synapsis, localizes to axis-associated chromatin in Arabidopsis and Brassica. J. Cell. Sci. 115: 3644-3655.

Bhatt, A. J., Lister, C., Page, T., Fransz, P., Findlay, K., Jones, G. H., Dickinson, H. G. and Dean, C. 1999. The DIF1 gene of Arabidopsis is required for meiotic chromosome segregation and belongs to the REC8/RAD21 cohesin gene family. Plant J. 19: 463-472.

Bickel, S. E. and Orr-Weaver, T. L. 1996. Holding chromatids together to ensure they go their seprate ways. BioEssays 18: 293-300.

Bione, N. C. P., Pagliarini, M. S. and Almeida, L. A. 2002a. An asynaptic mutation in soybean (Glycine max (L.) Merrill) associated with total absence of sister chromatid cohesiveness. Cytologia 67: 177-183.

- - - and - 2002b. A new and distinctive male-sterile, female-fertile desynaptic mutant in soybean (Glycine max). Hereditas 136: 97-103.

Dawe, R. K. and Cande, W. Z. 1995. The role of chromosome in maize meiotic spindle morphogenesis. J. Cell Biochem. (Suppl.) 21A: 438 .

Franklin, A. E., McElver, J., Sunjevaric, I., Rothstein, R. and Borwen, B. 1999. Three dimensional microscopy of the Rad 51 recombination protein during meiotic prophase. Plant Cell 11: 809-824.

Gottschalk, W. and Kleine, H. D. 1976. The influence of mutated genes on sporogenesis. A survey on the genetic control of meiosis in Pisum sativum. Theor. Appl. Genet. 48: 23-34.

— and Kaul, M. L. H. 1980a. Asynapsis and desynapsis in flowering plants. I. Asynapsis. Nucleus 23: 1-15.

— and - 1980b. Asynapsis and desynapsis in flowering plants. II. Desynapsis. Nucleus 23: 97-120.

Jauhar, P. P., Singh and Alice, C. J. 1971. Partial desynapsis in Pennisetum ramosum (Hochst.) Schweinf. Cytologia 36: 698-701. 
Ji, Y. E., Stelly, D. M., Donato, M. D., Goodman, M. M. and Williams, C. G. 1999. A candidate recombination modifier gene for Zea Mays L. Genetics 151: 821-830.

Koduru, P. R. K. and Rao, M. K. 1981. Cytogenetics of synaptic mutants in higher plants. Theor. Appl. Genet. 59: $197-214$.

Kumar, G. and Sharma, V. 2001. Induced desynapsis in Cicer Arietinum L. J. Cytol. Genet. 2(NS): 123-127.

— and Singh, V. 2002. Desynaptic variation in barley. J. Cytol. Genet. 3(NS): 127-131.

Maguire, M. P., Paredes, A. M. and Riess, R. W. 1991. The desynaptic mutants of maize as a combined defect of synaptonemal complex and chiasma maintenance. Genome 34: 879-887.

- - - and - 1993. Evidence from a maize desynaptic mutants points to a probable role of synaptonemal complex central region components in provision for subsequent chiasma maintenance. Genome 36: 797-807.

— 1993. Sister chromatid association at meiosis. Maydica 38: 93-106.

- 1995. Is the synaptonemal complex a disjunction machine? J. Hered. 86: 330-340.

Miyazaki, W. Y. and Orr-Weaver, T. L. 1994. Sister-chromatid cohesion in mitosis and meiosis. Ann. Rev. Genet. 28: 167-187.

Nasmyth, K., Peters, J. M. and Uhlmann, F. 2000. Splitting the chromosome: Cutting the ties that bind sister chromatids. Science 288: 1379-1384.

Palmer, R. G. and Horner, H. T. 2000. Genetics and cytology of a genic male-sterile mutant form a transposon-containing soybean population. J. Hered. 91: 378-383.

Peirson, B. N., Bowling, S. E. and Makaroff, C. A. 1997. A defect in synapsis causes male sterility in a T-DNA-tagged Arabdopsis thaliana mutant. Plant J. 11: 659-669.

Prakken, R. 1943. Studies of asynapsis in rye. Hereditas 71: 475-495.

Rao, K. G. R. and Kumar, O. A. 1983. Cytogenetics of a spontaneous desynaptic mutant in Chillies (Capsicum annum L). Cytologia 48: 195-199.

Rao, S. R. and Kumar, A. 2003. Cytological investigations in a desynaptic variant of Anogeissus sericea var. sericea, an important hardwood tree of Rajasthan. Bot. J. Linn. Soc. 142: 103-110.

Reddi, T. V. V. S. and Rao, D. R. M. 2000. Cytology of induced desynaptic mutant in rice Cytologia 65: 35-41.

Rees, H. 1961. Genotypic control of chromosome form and behavior. Bot. Rev. 27: 288-318.

Sharma, R. K. and Reinbergs, E. 1974. Cytogenetical analysis of a desynaptic mutant in barley. Cytologia 39: 77-81.

Simchen, G. and Stamberg, J. 1969. Fine and coarse controls of genetic recombination. Nature 222: 329-332.

Singh, S. and Gupta, P. K. 1981. Desynapsis in Zinnia haegeana L. Cytologia 46: 63-67.

Srivastava, H. M. 1974. Radiation induced desynaptic mutant in barley. Cytologia 39: 63-68. 